\title{
Proficient Car Parking System Based on Cluster Head Routing Protocol Utilizing IEEE 802.15.4
}

\author{
http://dx.doi.org/10.3991/ijoe.v12i06.5773 \\ Razi Iqbal ${ }^{1}$, Sharif Arif ${ }^{2}$ and H.H.R Sherazi ${ }^{3}$ \\ ${ }^{1}$ American University in the Emirates, Dubai, United Arab Emirates \\ 2 Lahore Leads University, Lahore, Pakistan \\ ${ }^{3}$ Telematics Lab, DEI, Politecnico di Bari, Bari, Italy
}

\begin{abstract}
The paper discusses a proposed model for car parking system based on cluster head routing protocol utilizing a low cost and power efficient communication technology, ZigBee (IEEE 802.15.4). The model is designed in a way that car parking is divided into different clusters and each cluster has a head which acts a messenger for transmitting information to other heads and the coordinator of the network. Each cluster head is a ZigBee Host (Router) which collects the information of car presence in the parking slot. This information is then passed to the coordinator of the network which is used to display the information of available parking slots in a specific car parking area. Since there is only one coordinator in the network, so heads can transmit information to the coordinator using multi-hop communication if direct communication is not possible. Several simulations were performed to gauge the efficiency of the proposed model, and results show that the proposed model is reliable in communication and efficient in its operation.
\end{abstract}

Index Terms-Cluster head routing protocol, wireless communication, power efficiency, ZigBee, multi-hop communication, automated parking system.

\section{INTRODUCTION}

World has now become a global village due to the new contraption in the field of information technology and the advancements let the researchers a step forward towards automation in all the aspect of life. Transportation industry has grown up tremendously around the world for last couple of decades with an improved standard of living particularly in the urban areas. Intelligent Transportation Systems (ITS) have grown significantly throughout the world in different way, forms and shape, e.g., car navigation systems, electronic toll collection, vehicle monitoring and surveillance and electronic car parking systems.

The specific area designated for the accumulation of the certain amount of vehicles, aimed at facilitating the car owners to park their cars, can be seen as a parking area and the area assigned to a single car in the overall parking area can be referred to as an available parking slot. Car parking area is the basic requirement of an automobile user interacting with the social environment. Proficient utilization of parking slots is also one of the major elements of concern due to increased vehicular density.

Due to the continuously mounting extent of automobiles, it has now become fairly complex to deal with the existing parking places in almost all the major cities across the globe. Inefficiency and lack of management of available parking areas are causing various problems to the society in the form of environment contamination and huge traffic jams yielding growing driver irritation. Expansion cost is also excessive to afford and owners are usually reluctant to adopt this way of increasing number of available parking slots.

Parking slots need to be adjusted in a way that enables the users to know the status of available spaces at the given moment. Various technologies have already been tested to obtain this goal $[1,5-8,12]$. Wireless sensor networks (WSNs) are the most promising technology being served for parking management system at the moment offering various advantages in terms of time saving and enables the automated parking to serve the customers efficiently. Therefore, automated parking management system are gaining popularity throughout the world.

Wireless sensor networks are advantageous in a way that they can be implemented in any existing car parking without the need of installing new apparatus for connectivity and electric supply to access each sensing device. It enables the authorities to avoid a lot of cost incurred on installing the infrastructure right from the scratch. For this reason, wireless sensor networks are also famous for their use across road-side car-parking. This technology is expected to provide an efficient and cost-effective solution towards the various parking problems that makes it possible for the parking owners to get the desired results with ease.

VDS \& VMS, SPARK, PGIS are some of the few parking systems currently implemented and all undergo the set of limitations of their own kinds. VDS \& VMS require standard frequency allocation, SPARKS have installation $\&$ maintenance problem which might disturb the normal parking operations and PGIS is not convenient for current parking lots [1]. The aim of the automation parking system is to efficiently assign the parking slots to the users and making the updated information available for the upcoming customers as well as for management staff.

The proposed model works efficiently by dividing the whole parking area into various clusters each having a cluster head responsible for communication with other heads. In this scenario, only the heads need to communicate current status of parking slots availability to the coordinator of the network. This makes it robust and reliable in a way that only a few nodes (heads) would communicate to coordinator instead of all the nodes present. It helps reducing the overhead and causes minimal congestion across the network yielding availability of real time information to the potential user when needed.

The remainder of this paper is organized as follows: The next section offers an overview of the current state of 
the art of ZigBee implementation using various technologies with respect to parking problems. Section III presents the proposed model for the proficient parking system based on the cluster head routing protocol implementing ZigBee as the communication Technologies. While the simulations are carried out to evaluate the performance of the proposed protocol and the results are discussed in section IV. Hence, the concluding remarks are finally stated in section $\mathrm{V}$.

\section{RELATED WORK}

Extensive research was proposed in the past to develop the efficient parking management system to facilitate driver in a way that driver can park its car without wasting extra time as well as avoiding congestion. ZigBee was introduced in 2004 and adopted widely across the globe soon after its inception due to its distinct features of lower power consumption and lower cost to build up the infrastructure [2].

Parking problems were massively noticed in big cities and instigated the significance of automated parking management system emphasizing on the fact that better management of existing parks is required rather than the increment in parking slots [3]. Almost $40 \%$ of traffic, causing congestion during peak hours, is the proportion which is unable to find an appropriate vacant parking slot [4].

There are number of techniques implemented for automation of parking areas. [5] presents one of them based on GSM technology named as Secure Parking Reservation System where a secret code is directly sent via SMS to driver's mobile phone who is looking for an empty slot and this code is used by the drivers while parking their cars and the same is reused when leaving the parking area. Smart signaling concept is also implemented in the parking management system in a way that ultrasonic sensor detects the presence of car in parking slot. If there is no car, green light is illuminated as a signal while red light indicates the non availability of a parking slot [6].

In [7], Sensors were replaced by image processing techniques based upon the brown rounded image captured in the parking area in order to sense the existence of vehicle in a parking slot. This technique gathers the pixels of reserved parking slots on the seven segmented display unit and extracts the empty parking slots for the drivers. [8] also used the same technique but they favor to use the already installed surveillance cameras in the parking area to fetch the images and process them comparing to a reference image of empty parking slot already constructed and given as the input. They deduce their results by calculating the ratio of vehicle to car-park pixels' areas.

Intelligent car parking system [9] was proposed using wireless sensors networks, where each parking slot is detected through sensors. This system is based on crossbow motes containing acoustic, temperature and light sensors. The authors discussed the way of detecting car presence by using these sensors. They also threw light on the advantages of using this technique which is majorly aimed at reducing the manpower and human effort. But this kind of extensive sensor usage increases the overall system cost against the level of efficiency that is also a major concern.

RFID based technology has been deployed in [10], named Smart Parking that consists of number of hardware devices like USB Cables, RFID reader, computers etc. to facilitate the check in and check out of the parking conveniently. To manage the record for number of busy and empty slots in the parking area, tracking information is saved in a central database while checking in and out without wastage of time for the convenience of users. Thanks to the idea of RFID based label identification, drivers would not have to stop on the check-outs for parking payments to avoid long queues usually seen at the parking exits.

Parking Guidance and Information System (PGIS) [11] was developed based upon sensor network which carries all the information from parking occupancy to control center where an information system has been installed responsible for capturing the data from ZigBee and guides the users accordingly as per their needs. It also provides the user with a secure mechanism for checking in and out in an easier way using RFID technology. The wireless connectivity is sought by the low power, cost effective and low data rate ZigBee that is quite reliable for this purpose.

Secure parking management and reservation system was proposed using ZigBee and GSM technology where users make their parking reservations using ZigBee technology. This system consists of a number of units working together for management and reservation of parking space. The entrance of parking consists of a GUI based display unit, showing the free parking slots for the users while entering in the parking area. Display unit is communicating via ZigBee devices to forward the data towards required destinations. They also introduced a security module where unauthorized access to vehicle would generate an SMS alert to the actual user and the system would not let the car to take exit from the parking area [12-13].

\section{PRoposed System MOdEL}

Figure 1 illustrates the proposed system model for the car parking system based on IEEE 802.15.4. As illustrated in the figure, each parking slot is installed with a sensor node which is a combination of a motion sensor and an indicator (LED or a small bulb) that indicates the availability of the parking slot, e.g., red for 'not available' and green for 'available'. Each sensor node is connected to a ZigBee module in such a way that one ZigBee module can accommodate more than 2 parking slots. As soon as the car is parked at the parking slot, motion sensor detects the availability of the car. The sensor node illuminates the indicator as red and the ZigBee module sends this information wirelessly to coordinator module which then sends a signal to the electronic gate that displays the total number of available car parking slots in that specific area.

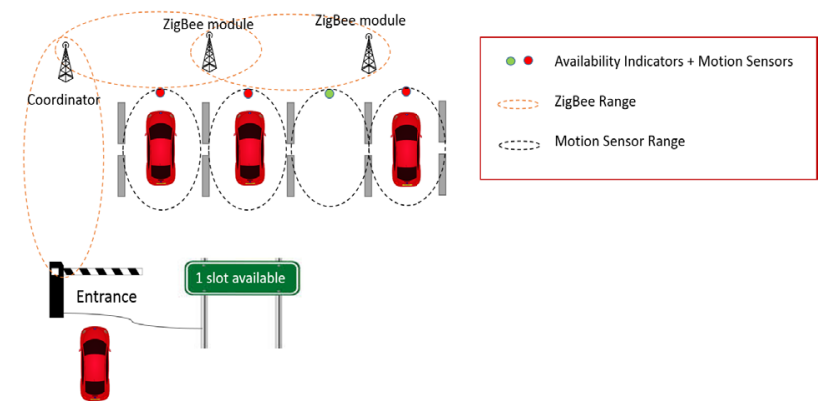

Figure 1. Proposed System Model 
The arrangement of ZigBee modules is made in such a way that each module is in the range of the other module based on ZigBee modules range which is around $100 \mathrm{~m}$. ZigBee communication supports various protocols, however, for this specific model, cluster head routing protocol is used in which each ZigBee module serves as a head for that specific cluster. Each cluster contains a head and some sensor nodes. Sensor nodes (motion sensors) in this case are connected to the ZigBee module using wires. Each cluster head is responsible for transmitting information to the coordinator about the availability of the parking slots. In case of cluster head not in range of the coordinator, multi-hop communication is done in which cluster heads send their information to nearby cluster head which then ultimately sends that information to the coordinator. Figure 2 shows multi-hop communication model in ZigBee using Cluster head routing protocol.

Fig. 2 illustrates 3 clusters A, B and C. Each cluster has some nodes and a head. Each cluster is arranged in such a way that cluster heads are in range of each other and they can communicate with their specific nodes as well as the neighbouring cluster heads. This cluster head communication helps in multi-hop communication, e.g., if head of cluster $\mathrm{C}$ wants to send some information to the coordinator which is out of its communication range; the information is first sent to head of cluster B, which sends the information to cluster A which ultimately sends this information to the coordinator.

\section{Simulation ReSUlts}

Several experiments were conducted to gauge the efficiency of the proposed model. Table I below shows the experimental data in which data was sent from one node to another based on measurement of time taken for communication between the nodes.

Table I illustrates the first set of experiments in which different sets of bytes of data was sent from heads to heads or heads to coordinator. As shown in the table, since the distance between the modules is kept constant at 10 feet, RSSI value remains stable between -62 to $-67 \mathrm{dBm}$. Due to small distance between the modules, no errors were detected as all the bytes were received from sender to receiver. As for the measurement of time, 15 bytes took $0.05 \mathrm{sec}, 50$ bytes took $0.09 \mathrm{sec}$ and 100 bytes took 0.14 $\mathrm{sec}$ of time for data communication.

Table II illustrates the experiments in which same arrangement is used except the distance which was increased from 10 feet to 20 feet.

As shown in Table II, with the increase in the distance between the modules, RSSI value fluctuated between -72 to $-79 \mathrm{dBm}$. However, due to small distance between the modules, no errors were detected as all the bytes were received from sender to receiver. As for the measurement of time, it remained the same as for the experiments conducted for 10 feet.

As shown in Table III, with the increase in the distance between the modules, RSSI value fluctuated between -72 to $-82 \mathrm{dBm}$. Just like the previous arrangement, even in this case all the bytes were received without any error. Furthermore, communication time also remained almost the same.

As shown in Table IV, with the increase in the distance between the modules, RSSI value fluctuated between -77 to $-86 \mathrm{dBm}$. All the bytes were received at the receiver

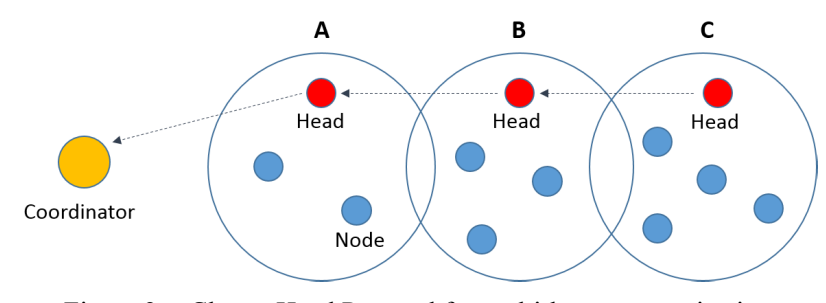

Figure 2. Cluster Head Protocol for multi-hop communication

TABLE I.

MEASUREMENT OF TIME TAKE FOR COMMUNICATION - I

\begin{tabular}{|c|c|c|c|c|}
\hline Distance & No. of Bytes & $\begin{array}{c}\text { Time } \\
\text { (sec) }\end{array}$ & $\begin{array}{c}\text { RSSI } \\
\mathbf{( d B m )}\end{array}$ & $\begin{array}{c}\text { Bytes Re- } \\
\text { ceived }\end{array}$ \\
\hline \multirow{3}{*}{10 feet } & 15 & 0.05 & -62 & 15 \\
\cline { 2 - 5 } & 50 & 0.09 & -67 & 50 \\
\cline { 2 - 5 } & 100 & 0.14 & -65 & 100 \\
\hline
\end{tabular}

TABLE II.

MEASUREMENT OF TIME TAKE FOR COMMUNICATION - II

\begin{tabular}{|c|c|c|c|c|}
\hline Distance & No. of Bytes & $\begin{array}{c}\text { Time } \\
\text { (sec) }\end{array}$ & $\begin{array}{c}\text { RSSI } \\
\text { (dBm) }\end{array}$ & $\begin{array}{c}\text { Bytes Re- } \\
\text { ceived }\end{array}$ \\
\hline \multirow{3}{*}{20 feet } & 15 & 0.05 & -72 & 15 \\
\cline { 2 - 5 } & 50 & 0.09 & -77 & 50 \\
\cline { 2 - 5 } & 100 & 0.14 & -79 & 100 \\
\hline
\end{tabular}

TABLE III.

MEASUREMENT OF TIME TAKE FOR COMMUNICATION - III

\begin{tabular}{|c|c|c|c|c|}
\hline Distance & No. of Bytes & $\begin{array}{c}\text { Time } \\
(\mathbf{s e c})\end{array}$ & $\begin{array}{c}\text { RSSI } \\
(\mathbf{d B m})\end{array}$ & $\begin{array}{c}\text { Bytes Re- } \\
\text { ceived }\end{array}$ \\
\hline \multirow{3}{*}{30 feet } & 15 & 0.05 & -72 & 15 \\
\cline { 2 - 5 } & 50 & 0.08 & -77 & 50 \\
\cline { 2 - 5 } & 100 & 0.15 & -82 & 100 \\
\hline
\end{tabular}

TABLE IV.

MEASUREMENT OF TIME TAKE FOR COMMUNICATION - IV

\begin{tabular}{|c|c|c|c|c|}
\hline Distance & No. of Bytes & $\begin{array}{c}\text { Time } \\
\text { (sec) }\end{array}$ & $\begin{array}{c}\text { RSSI } \\
(\mathbf{d B m})\end{array}$ & $\begin{array}{c}\text { Bytes Re- } \\
\text { ceived }\end{array}$ \\
\hline \multirow{3}{*}{40 feet } & 15 & 0.05 & -86 & 15 \\
\cline { 2 - 5 } & 50 & 0.10 & -77 & 50 \\
\cline { 2 - 5 } & 100 & 0.15 & -82 & 100 \\
\hline
\end{tabular}

TABLE V.

MEASUREMENT OF TIME TAKE FOR COMMUNICATION - V

\begin{tabular}{|c|c|c|c|c|}
\hline Distance & No. of Bytes & $\begin{array}{c}\text { Time } \\
(\mathbf{s e c})\end{array}$ & $\begin{array}{c}\text { RSSI } \\
(\mathbf{d B m})\end{array}$ & $\begin{array}{c}\text { Bytes Re- } \\
\text { ceived }\end{array}$ \\
\hline \multirow{3}{*}{50 feet } & 15 & 0.06 & -86 & 15 \\
\cline { 2 - 5 } & 50 & 0.09 & -85 & 50 \\
\cline { 2 - 5 } & 100 & 0.18 & -89 & 100 \\
\hline
\end{tabular}

without any significant errors. Time in this case jumped to $0.10 \mathrm{sec}$ for 50 bytes, however, it remained same for 15 and 100 bytes.

As shown in Table $\mathrm{V}$, with the increase in the distance between the modules, RSSI value fluctuated between -85 to $-89 \mathrm{dBm}$. All the bytes were received at the receiver without any significant errors. In this case communication time was also almost the same.

The above conducted experiments showed excellent results as far as data reliability is concerned; data was received at receiver without any errors in all the experi- 
ments, regardless of increase in distance. Similarly, the communication time was also in control as it was under 1 sec for transfer of information of around 100 bytes at distance of even 50 feet.

Although the experiments showed excellent results, still some more experiments were conducted to gauge the efficiency of the proposed system in multi-hop communication.

Table VI shows data of experiment conducted to measure the time taken by the data from sender (head) to receiver (coordinator) with 2 hops. Data is encouraging in a sense that it is still under $1 \mathrm{sec}$ for even 100 bytes.

Table VII shows data of experiment conducted to measure the time taken by the data from sender (head) to receiver (coordinator) with 3 hops. Even with 3 hops, data was transmitted with good pace.

The experiments conducted were to check the efficiency of ZigBee in a multi-hop outdoor environment. Different set of experiments showed excellent results for communication time and reliability of data. Even with large amount of bytes (100 bytes) the data showed no loss. Furthermore, even with the increase in distance data was transmitted in reasonable time without any problems.

\section{CONCLUSION}

The model for an automated car parking system was presented in this work based upon the novel approach of cluster head routing protocol using ZigBee as the communication technology where overall parking area was divided into smaller clusters each having a cluster head (ZigBee module) and few sensor nodes (motion sensors) equipped with an indicator on every parking slot. Motion sensors in a cluster detect the presence or absence of vehicles in the parking slots and communicate the information only to their cluster head which is further responsible for communicating this information to the parking coordinator. The communication is even possible through peer heads (multi-hop) if the coordinator is not in the direct range of a cluster head to convey the real time information displayed to drivers on the outer display unit. Various experiments were conducted to cross check the proposed approach regarding the performance and efficiency of the system which clearly demonstrate the strength, reliability and real time accuracy of the information based on the proposed model.

\section{REFERENCES}

[1] A Comparative Study of Different Smart Parking Assist Systems Using Wireless Sensor Networks Vivekanand P. Thakare\&NekitaChavan G. H. Raisoni College of Engineering, Digdoh hills, Wanadongari, Nagpur International Journal of Smart Sensors and Ad Hoc Networks (IJSSAN) ISSN No. 2248-9738 Volume-1, Issue-4, 2012

[2] Huang, H. C., Huang, Y. M., \& Ding, J. W. (2006, January). An implementation of battery-aware wireless sensor network using ZigBee for multimedia service. In Consumer Electronics, 2006. ICCE'06. 2006 Digest of Technical Papers. International Conference on (pp. 369-370). IEEE.

[3] K. Axhausen and J. Polak, "Choosing the type of parking: stated preference experiments in the UK and Germany," Working Paper, Transport Studies Unit, Oxford University, p. 253, 1990.
TABLE VI.

MEASUREMENT OF TIME TAKE FOR MULTI-HOP COMMUNICATION - I

\begin{tabular}{|c|c|c|c|c|}
\hline Distance & No. of Bytes & $\begin{array}{c}\text { No. of } \\
\text { hops }\end{array}$ & Time (sec) & $\begin{array}{c}\text { Bytes Re- } \\
\text { ceived }\end{array}$ \\
\hline \multirow{3}{*}{50 feet } & 15 & 2 & 0.17 & 15 \\
\cline { 2 - 5 } & 50 & 2 & 0.31 & 50 \\
\cline { 2 - 5 } & 100 & 2 & 0.74 & 100 \\
\hline
\end{tabular}

TABLE VII.

MEASUREMENT OF TIME TAKE FOR MULTI-HOP COMMUNICATION - II

\begin{tabular}{|c|c|c|c|c|}
\hline Distance & No. of Bytes & $\begin{array}{c}\text { No. of } \\
\text { hops }\end{array}$ & Time (sec) & $\begin{array}{c}\text { Bytes Re- } \\
\text { ceived }\end{array}$ \\
\hline \multirow{3}{*}{50 feet } & 15 & 3 & 0.33 & 15 \\
\cline { 2 - 5 } & 50 & 3 & 0.49 & 50 \\
\cline { 2 - 5 } & 100 & 3 & 0.91 & 100 \\
\hline
\end{tabular}

[4] P. White, No Vacancy: Park Slopes Parking Problem And How to Fix It. Transalt.org, 2007.

[5] R. Yusnita and N. Fariza, "A secure parking reservation system using GSM technology," International Journal of Computer and Communication Engineering, vol. 2, no. 4, July 2013.

[6] K. Amin, M. Norli, L. Pakapan, and K. Pantea, "Smart parking system (SPS) architecture using ultrasonic detector," International Journal of Software, Engineering and Its Applications, vol. 6, no. 3, 2012

[7] R. Yusnita, N. Fariza, and B. Norazawinati, "Intelligent parking space detection system based on image processing," International Journal of Innovation, Management and Technology, vol. 3, no. 3, June 2012.

[8] S. Funck, N. Mohler, and W. Oertel, "Determining car- park occupancy from single images," in Proc. the IEEE Intelligent Vehicles Symposium University of Parma, Italy, June 2004, pp. 1417. http://dx.doi.org/10.1109/ivs.2004.1336403

[9] ZigBee/IEEE 802.15.4 Summary SinemColeriErgen Email: csinem@eecs.berkeley.edu September 10, 2004

[10] Zeydin PALA* and Nihat INAN;**,.Smart Parking Applications Using RFID Technology. Jin Wen University of Science and Technology. April 2009

[11] Patil, M., \& Bhonge, V. N. (2013, April). Parking Guidance And Information System Using RFID And ZigBee. In International Journal of Engineering Research and Technology (Vol. 2, No. 4 (April-2013)). ESRSA Publications.

[12] A. Sayeeraman and P. S. Ramesh, "ZigBee and GSM based secure vehicle parking management and reservation system," Journal of Theoretical and Applied Information Technology, vol. 37, no. 2, 2012.

[13] M. R. M. Kassim, "Design, development and implementation of smart home system using RF and power line communication" in Proc. 2nd National Intelligent Systems and Information Technology Symposium (ISITS'07), vol. 3, no. 3, pp. 200-208, 1986.

\section{AUTHORS}

Razi Iqbal is with American University in the Emirates, Dubai, United Arab Emirates (razi.iqbal@aue.ae).

Sharif Arif is with Lahore Leads University, Lahore, Pakistan (shariif.arif@gmail.com).

H.H.R Sherazi is with the Telematics Lab, DEI, Politecnico di Bari, Bari, Italy (sherazi@poliba.it).

Submitted 17 April 2016. Published as resubmitted by the authors 23 May 2016. 